\title{
Adolescents and Emerging Adults with Chronic Kidney Disease: Their Unique Morbidities and Adherence Issues
}

\author{
Maria E. Diaz-Gonzalez de Ferris \\ UNC Kidney Center, Chapel Hill, N.C., USA
}

\section{Key Words}

Adherence $\cdot$ Health care transition - Chronic kidney disease . End-stage kidney disease

\begin{abstract}
Medical advances have increased the survival rates of adolescents and emerging adults with chronic kidney disease and end-stage kidney disease. Patients in these two age groups share many characteristics: their brain will fully develop in their late 20s; they have cognitive impairment related to disease severity and duration, are at greater risk of nonadherence, and need to learn to manage their complex medical condition and comorbidities while trying to achieve independence from their parents. The medical and psychosocial characteristics of these patients are discussed, and possible strategies for their care suggested.
\end{abstract}

Copyright $\odot 2011$ S. Karger AG, Basel

\section{Introduction}

Several centuries ago, adolescence was defined as the period between childhood and adulthood, typically between the ages of 12 and 18 years. However, emerging adulthood is a newer theoretical term coined by Dr. J. Arnett. This developmental time period was created to capture the developmental characteristics of people aged 18-
29 years. Conceptually, emerging adulthood has five proposed themes: identity explorations, instability, self-focus, feeling 'in-between' and possibilities. The themes incorporated into the emerging adult theory recognize that during the last 50 years, a shift has occurred and people have begun to postpone marriage and parenthood until their late twenties. Now, individuals are spending their late teens through their mid-20s in self-focused exploration through intimate relationships and career development. While the line between adolescence and emerging adulthood is not clear, patients with chronic kidney disease (CKD) or end-stage kidney disease (ESKD) in both age groups may share similar characteristics, comorbidities, and challenges.

In this article, I will use the terms adolescents (teens) and emerging adults as 'TEA', discuss the epidemiological data of this population, their unique challenges and morbidities, and will suggest health provider strategies for their care.

\section{Epidemiological Data on Adolescent and Emerging Adult Patients with CKD/ESKD}

The prevalence of CKD in TEA patients is unknown, and most ESKD registries in the world provide no incidence or prevalence data on patients in these combined age group. ANZDATA is the one ESKD registry that in-

\section{KARGER}

Fax +41613061234 E-Mail karger@karger.ch www.karger.com

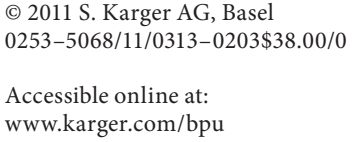


Fig. 1. Living kidney donation by age group in the USA (1988-2010). Source: http://optn.transplant.hrsa.gov/latestDa ta/rpt.Data.asp.

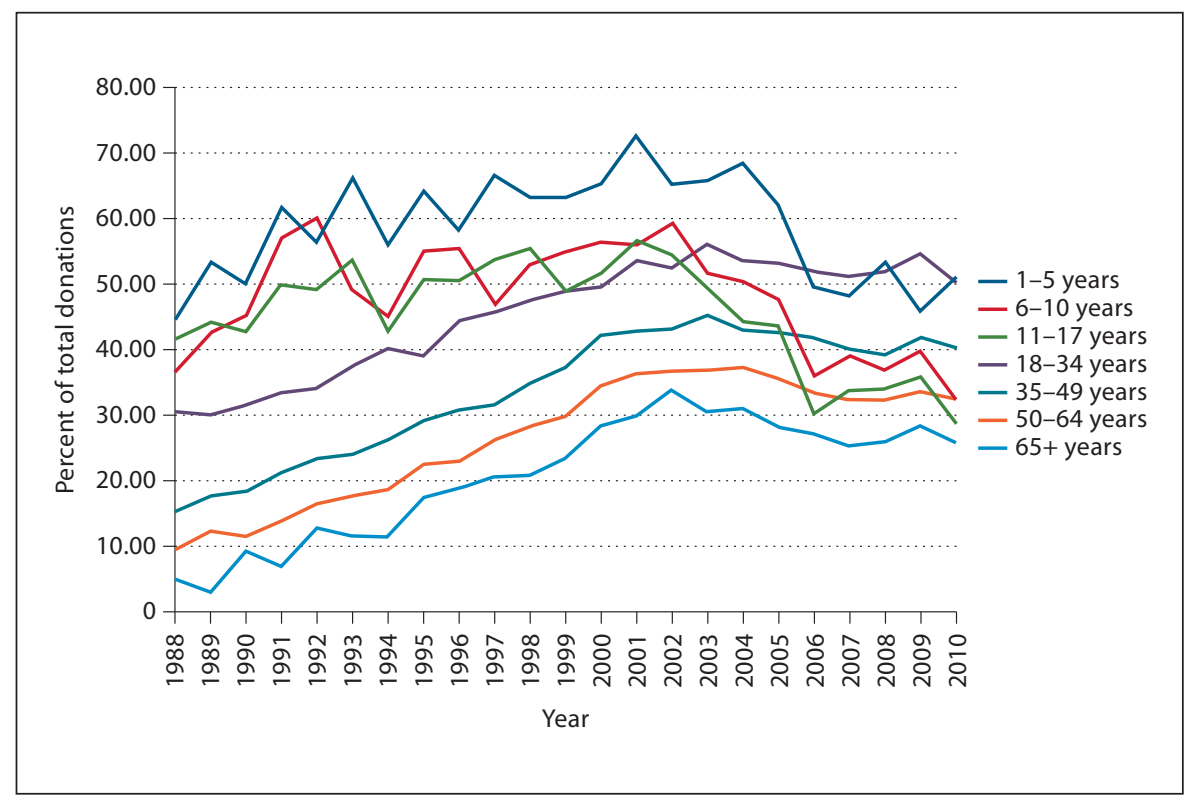

cludes 15- to 24-year-old patients, reporting that there are at least 7,000 adolescent/emerging-adult dialysis patients and 11,000 renal transplant recipients in the EU, North America, Australia, and New Zealand [1].

The 10-year survival rate for adolescents with ESKD has increased during the last 25 years from 70 to $85 \%$ [2], and is greater than the 10-year survival for adults with ESKD, which is $50 \%$. This greater survival among TEA patients with ESKD is likely due to changes in treatment such as improved nutrition, erythropoiesis-stimulating agents, safer renal biopsy procedures, improved hemo-/ peritoneal dialysis equipment (and prescriptions), and transplant therapy. The improved medical treatments have not only increased the survival rates of those with ESKD, but have also influenced transplant procedures. In fact, adolescent transplant recipients have a better 10-year survival rate compared to dialysis patients. While the survival rate has improved, the life expectancy for those on dialysis is approximately 25 and $40 \%$ for transplant recipients when compared to age-matched peers [3]. Unfortunately, in the USA health disparities are present in adolescent-onset ESKD as patients of minority groups have a lower chance of being transplanted or retransplanted [2].

\section{Chronic Renal Replacement Treatment Modality}

Most TEA patients with ESKD undergo hemodialysis as the renal replacement treatment modality [3]. In the USA, TEA patients on hemodialysis are managed at di- alysis units directed by adult-focused internal medicine (IM) nephrologists, and these providers have a different ESKD practice and training than pediatric nephrologists [4]. In a random national sample of the USA, 17\% of adolescents cared for by IM nephrologists received peritoneal dialysis, and pediatric nephrologists were $60 \%$ more likely than IM nephrologists to recommend peritoneal dialysis for identical patients (odds ratio, 1.61; 95\% confidence interval, 1.35-1.92). This was true regardless of dialysis training, years in practice, practice setting, geography, or patient characteristics [4]. In general, pediatric nephrologists avoid hemodialysis because when compared to peritoneal dialysis, hemodialysis has a greater negative impact on quality of life, prevents school/work attendance, decreases academic achievement, and lowers the opportunity to find fulltime jobs. Additionally, as the median age of dialysis patients in these IM practices is 64 years [3], and the TEA patients in these units account for only $3 \%$ of the census, it is difficult for them to find peers and socialization opportunities.

Compared to all age groups, TEA patients with ESKD receive kidney transplants at high rates (adolescents have the highest rates). While living-donated kidney transplantation has the best patient and graft outcomes, a concerning decline (steepest among the 11- to 17-year-old recipients) in living donors has been noted in most age groups in the USA, likely reflecting the changes in organ allocation preferential to this age group (see fig. 1). 
Table 1. Age at diagnosis of CKD/ESKD and patient characteristics

\begin{tabular}{lll}
\hline & $\begin{array}{l}\text { Infants and } \\
\text { children }\end{array}$ & TEA \\
\hline Cause of CKD/ESKD & congenital anomalies & glomerular disease \\
Duration of disease & longer & shorter \\
Surgical procedures of hospitalizations & more & less \\
Hypertension & less & more \\
Immunosuppression & no & yes \\
Fragile child syndrome & more & less \\
\hline
\end{tabular}

\section{Unique Challenges and Morbidities of TEA Patients with CKD/ESKD}

TEA patients with CKD/ESKD pose unique challenges to health care providers and to the health care system as a whole. Care must address not only the disease itself, but also the additional challenges and comorbidities unique to the adolescent patients and their families discussed below:

(1) A developing brain. The brain in healthy adolescents and emerging adults completes its development process in the mid- to late 20s. The last step of development is located in the prefrontal cortex area, responsible for 'executive brain functions' such as foresight, planning, evaluation of risk and reward.

(2) Lower socialization/self-esteem. Long-term medical and psychosocial outcomes of pediatric-onset ESKD patients have been published on transplant recipients [59] including lower employment rate and life accomplishments (marriage or having children).

(3) Nonadherence to treatment. TEA patients with $\mathrm{CKD} / \mathrm{ESKD}$ are at greater risk for nonadherence to treatment compared to other age groups. Further, nonadherence is the number one cause of kidney graft failure in this transition age group. With every episode of rejection, graft survival decreases. Contradictory findings have been reported by programs in the UK and Canada on TEA patients with a kidney transplant who have transferred to adult-focused services. While Watson [10] reported a $40 \%$ transplant loss in TEA patients of a British cohort of 20 patients, Koshy et al. [11] showed no increase in graft loss in a Canadian cohort of 115 TEA patients. Adherence among adolescents also is compromised by both poor understanding and poor consequence recognition, leading to inconsistent commitment to the treatment regimen. Knowledge of medical concepts and management of pediatric medical con- ditions is related strongly to literacy levels of patients and their caregivers. Unfortunately, there are no valid instruments or tools that measure adherence in this age group.

(4) Independence from parent-directed care. Health care transition process refers to a purposeful, planned process in which TEA patients assume increasing responsibility for their health condition management. A successful health care transition process includes proficiency in knowledge of the medical condition and medications, as well as developed self-direction and self-activation skills; all of which affect treatment adherence and patient outcomes. The parental role in successfully relinquishing care is likely related to family characteristics, level of parental distress, and worry about the child's health. Parents of chronically ill children have higher marital distress and decreased marital harmony when compared with parents of healthy children.

(5) Age at diagnosis of CKD/ESKD. Younger patients have congenital anomalies of the kidney and urinary tract which may be diagnosed even in utero, whereas in older patients CKD/ESKD is likely of glomerular etiology [2]. The so-called fragile child syndrome may be more pronounced in these families.

(6) Type of diagnosis. TEA patients diagnosed during infancy or childhood have congenital anomalies of the kidneys and urinary tract as the etiology of their CKD/ ESKD. Enuresis, multiple surgical interventions, hospitalizations during childhood and the need for self-catheterizations are more prevalent in this younger age group, impairing socialization and peer acceptance. Adolescentonset CKD/ESKD has unique comorbidities and psychological issues with the need for steroids, immunosuppressant and antihypertensive medications (table 1).

(7) Multiple roles. TEA patients with CKD/ESKD have to cope with their medical condition as they seek an identity, peer recognition and independence from their par- 
Fig. 2. Age-standardized heights and weights in pediatric hemodialysis patients, USA 2007. Source: USRDS http://www. usrds.org/2009/view/v2_08_peds.asp.

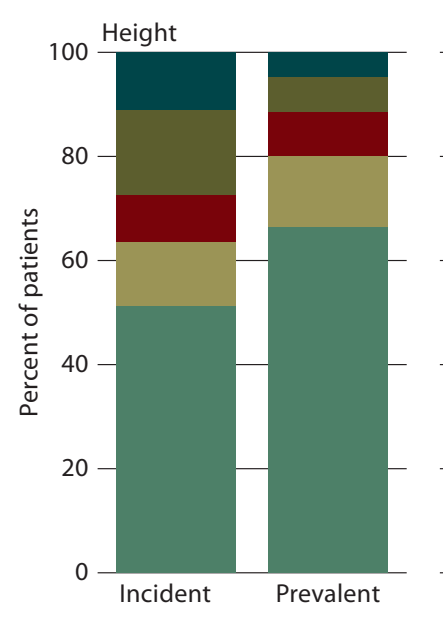

Weight

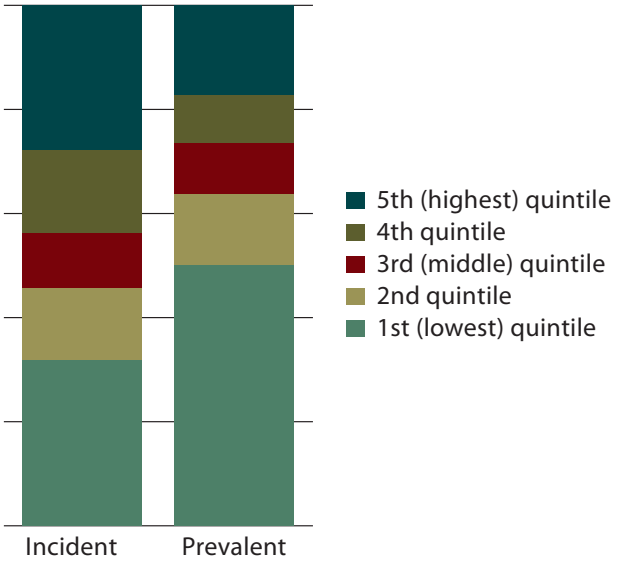

ents/caregivers, while transitioning from pediatric to IM nephrology practices.

(8) Physical appearance. Dysmorphic features in many instances result in a lower quality of life. One of the most important medical predictors of health-related quality of life (HRQOL) is growth. Short stature for TEA patients with CKD decreases their HRQOL as adults. Figure 2 depicts the distribution of pediatric patients by height, as determined from 2007 dialysis service claims for the adjustment of payments in the USA. In the incident pediatric population, $52 \%$ of patients are in the lowest height quintile of the general population, while $32 \%$ are in the lowest weight quintile. In the prevalent population, $66 \%$ of patients are in the lowest height quintile of the general population and $50 \%$ are in the lowest weight quintile (fig. 2). Despite these facts, there is low use of growth hormone in the USA, likely related to insurance coverage.

(9) Vascular calcification, coronary heart disease and sudden death. Cardiovascular disease is a leading cause of their morbidity and mortality; for ESKD patients aged 15-19 years, cardiovascular event rates are nearly 1,000fold higher than in their age-matched peers, while the mortality rate for those aged 25-34 years is similar to that of 75 -year-olds in the general population.

(10) Impaired cognition. TEA patients with CKD/ ESKD with longer disease duration or worse renal function will have greater cognition deficits [12-15]. Multiple hospitalizations and school absenteeism (particularly if diagnosed at an early age) will also impact the ability to have better employment opportunities.

\section{Provider Issues}

Currently, the pediatric nephrology workforce is not meeting the multifaceted demand of TEA patients with CKD/ESKD. In fact, there are three states in the USA with no pediatric nephrologist, so issues such as health care transition or quality of life often get less attention than other medical issues. Most pediatric nephrologists are in academic settings, with easier access to a multidisciplinary team compared to IM nephrologists. As TEA patients with CKD/ESKD are complex with technology and medication dependence, finding a primary care physician who feels comfortable taking care of these patients is at times difficult. Therefore, by default the pediatric nephrology practices become the medical homes of these patients and well adolescent visits are neglected, perhaps accounting for their low immunization rates [16].

At the 2005 American Society of Pediatric Nephrology Meeting during a symposium addressing transition, the audience expressed many concerns when negotiating when and how to transfer a pediatric patient to adult care such as: 'At times, we have difficulty finding an internist who will accept our patients.' 'We are concerned that our patients will have less monitoring because of the greater number of adult-onset kidney patients with worse comorbidities.' 'We keep the patients under our care until they are medically stable.' 'Is the recommended age to begin transition at 14 years of age appropriate for our patients who may have impaired cognition?' 'We have pa- 
Table 2. Strategies to help TEA with CKD

Trust building through private conversations

Empathize with them, honoring their disabilities and resilience

Engage and lead them to successful disease self-management

Nurture their positive assets to achieve adherence and good

outcomes tients in their 30s who will not transfer.' 'We have difficulty admitting patients at a children's hospital after 16 years of age.' Many of these concerns may stem from pediatric nephrologists not being clear on the level of care their patients will receive once transferred to IM nephrology practices.

\section{IM Nephrologists}

IM nephrologists traditionally work in private practice where they have less access to multidisciplinary team members. Their TEA patients are few compared to adultonset patients ( $<3 \%$ of their practice) and 'come with parents'. IM nephrologists may not be as familiar with developmental issues and rare diseases such as Lowe's and cystinosis. Additionally, insurance coverage may be an important factor, as the pediatric-onset patient may lose coverage from their parents insurance after the age of 19, unless they are full-time students. Lastly, adult-focused nephrologists may have less experience and exposure to TEA patients and their age specific challenges and unique morbidities.

\section{Other Health Providers and Family Members}

A successful health care transition process requires not only the participation of motivated TEA patients and their families, but also interdisciplinary collaboration. In fact, the literature on health care transition has had representation of health providers in several health disciplines, reflecting the importance of this topic to all involved in these patients' care $[17,18]$. The role of the family in preparing their TEA patients with chronic medical conditions to a successful transition to adult-focused care is critical, but having a child with a chronic illness places tremendous stressors on the family $[19,20]$. Siblings may miss out on activities and emotional support because the parents are immersed in the care of the child with CKD or ESKD [8]. High family cohesiveness and low family conflict correlate with fewer child behavior problems and lower internalizing symptoms, suggesting that a welladjusted supportive family can help the child cope with CKD more successfully [21, 22]. Lastly, the role of the school, community and peers in the area of health care transition remains to be elucidated.

\section{Strategies for the Care of TEA Patients with CKD}

Based on the American Academy of Pediatrics, the following strategies for the care of TEA patients with CKD should be considered: build the patient's trust by ensuring privacy in all communication. If the TEA patient comes into the room with their parent, start the interview with everyone in the room, but then ask the parents to leave. Be aware that TEA patients who were diagnosed with CKD/ESKD at a younger age may have had more socialization challenges than patients who developed CKD/ESKD later in life. Focus on the patient's positive characteristics with an ultimate aim to help build resilience, better treatment adherence, and improved health outcomes. Mention that the many challenges associated with having CKD may be minimized by having a supportive family, positive social peer support and engaging in activities that take away their selffocus such as volunteering in community activities. Empathize with them honoring their disabilities and resilience. Engage and lead them to successful disease selfmanagement. Encourage TEA patients to ask questions, learn about their illness, and take personal responsibility. As many adolescents may be missing significant portions of school due to their illness, encourage families to ask schools for accommodations and supplemental support or an individualized education plan. Nurture their positive assets to achieve adherence and good outcomes. Design a planned, interdisciplinary approach to the care of these patients with the goals and objectives clearly delineated for all in a culturally and literacy appropriate format. I offer the following mnemonics to remember these strategies (see table 2).

\section{Conclusions}

TEA patients with CKD/ESKD have a wide range of medical and psychosocial factors that include: medical/ physical concerns, psychosocial functioning, long-term disabilities, multiple hospitalizations, health care transi- 
tion, impaired cognition, dietary restrictions, treatment adherence, and comorbidities such as coronary heart disease, anemia, hypertension, vascular calcification and metabolic bone disease. It is important to determine the age at diagnosis and etiology of CKD/ESKD patients as this will guide their care. Understanding their family background will also suggest approaches to optimize their care. It is imperative that valid information tools are developed to measure the health transition process and treatment adherence of these patients. Care strategies have been suggested.

\section{Acknowledgements}

The author wishes to thank Miss Nicole Fenton, MS, and Mr. Bradley Layton for their insightful comments and suggestions.

\section{References}

1 Australia and New Zealand Dialysis and Transplant Registry Report 2009. http:// www.anzdata.org.au/vl/report_2009.html; accessed July 27, 2010.

- 2 Ferris ME, Gipson DS, Kimmel PL, Eggers PW: Trends in treatment and outcomes of survival of adolescents initiating end-stage renal disease care in the United States of America. Pediatr Nephrol 2006;21:10201026.

3 USRDS 2009 Annual Data Report: Atlas of Chronic Kidney Disease and End-Stage Renal Disease in the United States. http://www. usrds.org/adr.htm; accessed August 15, 2010.

4 Furth SL, Hwang W, Yang C, Neu AM, Fivush BA, Powe NR: Relation between pediatric experience and treatment recommendations for children and adolescents with kidney failure. JAMA 2001;285:1027-1033.

-5 Bartosh SM, Leverson G, Robillard D, Sollinger HW: Long-term outcomes in pediatric renal transplant recipients who survive into adulthood. Transplantation 2003;76: 1195-1200.

-6 Furr LA: Psycho-social aspects of serious renal disease and dialysis: a review of the literature. Soc Work Health Care 1998;27:97118.

7 McEnery PT, Alexander SR, Sullivan K, Tejani A: Renal transplantation in children and adolescents: the 1992 annual report of the North American Pediatric Renal Transplant Cooperative Study. Pediatr Nephrol 1993;7: 711-720.
8 Groothoff JW, Grootenhuis M, Dommerholt A, Gruppen MP, Offringa M, Heymans HS: Impaired cognition and schooling in adults with end stage renal disease since childhood. Arch Dis Child 2002;87380-87385.

-9 Broyer M, Le Bihan C, Charbit M, Guest G, Tete MJ, Gagnadoux MF, et al: Long-term social outcome of children after kidney transplantation. Transplantation 2004;77:10331037.

10 Watson AR: Problems and pitfalls of transition from paediatric to adult renal care. Pediatr Nephrol 2005;20:113-117.

11 Koshy SM, Herbert D, Lam K, Stukel TA, Guttmann A: Renal allograft loss during transition to adult healthcare services among pediatric renal transplant patients. Transplantation 2009;87:1733-1736.

$>12$ Gipson DS, Hooper SR, Duquette PJ, Wetherington CE, Stellwagen KK, Jenkins TL, et al: Memory and executive functions in pediatric chronic kidney disease. Child Neuropsychol 2006;12:391-405.

13 Groothoff JW, Grootenhuis MA, Offring M, Stronks K, Hutten GJ, Heymans HSA: Social consequences in adult life of end-stage renal disease in childhood. J Pediatr 2005; 146:512-517.

14 Brouhard BH, Donaldson LA, Lawry KW, McGowan KR, Drotar D, Davis I, Rose S, Cohn RA, Tejani A: Cognitive functioning in children on dialysis and post-transplantation. Pediatr Transplant 2000;4:261-267.
15 Bawden HN, Acott P, Carter J, Lirenman D, MacDonald GW, McAllister M, McDonnell MC, Shea S, Crocker J: Neuropsychological functioning in end-stage renal disease. Arch Dis Child 2004;89:644-647.

16 National Institute of Diabetes and Digestive and Kidney Diseases: USRDS 2007 Annual Data Report: Atlas of Chronic Kidney Disease and End-Stage Renal Disease in the United States. Bethesda, National Institutes of Health, 2007.

17 Reiss J, Gibson R: Health care transition: destinations unknown. Pediatrics 2002;110: 1307-1314.

18 Betz CL: Transition of adolescents with special health care needs: review and analysis of the literature. Issues Compr Pediatr Nurs 1974;27:179-241.

19 Tew BJ, Payne H, Laurence KM: Must a family with handicapped child be a handicapped family? Dev Med Child Neurol 1974;16 (Suppl 32):95-98.

20 Lansky SB, Cairns NU, Hassanein R, Wehr J, Lowman JT: Childhood cancer: parental discord and divorce. Pediatrics 1978;62:184188.

21 Soliday E, Kolon TF, Lande MB: Psychosocial adjustment in children with kidney disease. J Pediatr Psychol 2000;25:93-103.

22 Soliday E, Kool E, Lande MB: Family environment, child behavior, and medical indicators in children with kidney disease. Child Psychiatry Hum Dev 2001;31:279-295. 